\title{
La médecine ambulatoire de demain, victime du financement d'hier
}

\author{
Jürg Schlup \\ Dr méd., président de la FMH
}

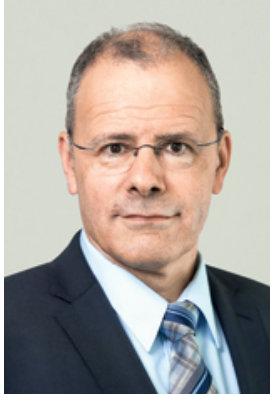

A nouveau, l'avenir de la médecine ambulatoire est en péril. Après une première intervention du Conseil fédéral dans le tarif, jugée illégale par le Tribunal cantonal de Lucerne car inappropriée et motivée par des considérations politiques, une deuxième intervention se profile. La FMH dans sa réponse à la consultation mardi dernier a pris clairement position en faveur d'un tarif juste et de l'autonomie tarifaire, et donc pour une médecine ambulatoire forte, mais il est impossible de savoir si nos arguments seront entendus: en effet, la politique de la santé se contente d'un activisme ponctuel en ciblant les primes et en s'éloignant toujours plus d'une conception rationnelle du système dans son ensemble. Comme chacun sait - Conseil fédéral compris -, «l'augmentation de la part de soins ambulatoires tend à entrấner une augmentation de la part des primes dans le financement du système de santé» [1], le secteur ambulatoire étant financé à $100 \%$ par les primes, alors que le sec-

\section{Les plafonds budgétaires ne présentent aucun avantage en termes de coûts.}

teur hospitalier est en grande partie financé par l'impôt cantonal. Par conséquent, le transfert de l'hospitalier vers l'ambulatoire réduit certes les coûts mais génère une hausse des primes. Notre priorité politique absolue devrait donc porter sur le financement, car ce n'est qu'avec un financement uniforme des prestations ambulatoires et hospitalières que le développement du secteur ambulatoire pourra réduire les coûts sans déclencher une hausse des primes.

Mais comme les cantons sont plutôt satisfaits de voir leurs charges diminuer grâce aux assurés, on peut douter qu'une telle réforme soit en mesure d'obtenir une majorité politique - d'où un potentiel d'économie de l'ordre du milliard [2] inexploité à ce jour. On préfère combattre la hausse des primes en limitant les prestations ambulatoires, avec des conséquences douloureuses pour les patients et les médecins. La réduction de 700 millions visée actuellement par l'intervention tarifaire témoigne du même activisme que celui déployé pour limiter les installations et surtout en voulant instaurer un plafond budgétaire. Or les partisans de ce dernier point semblent ignorer qu'une limitation
Total des dépenses de santé en \% du PIB [4].

12 Aucun avantage d'un plafond budgétaire

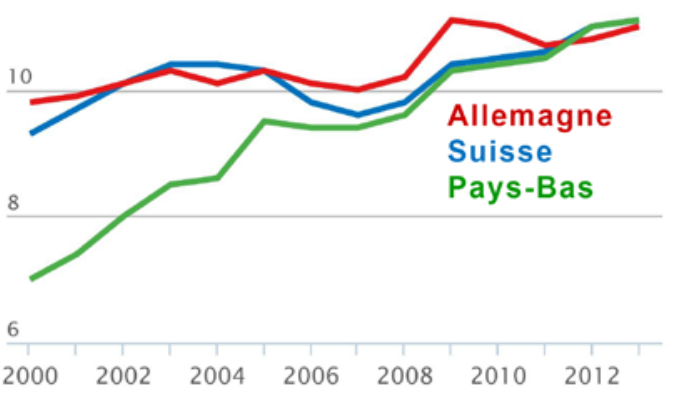

des coûts aura forcément un impact sur les prestations. Pour rassurer les assurés, on affirme que $20 \%$ des prestations sont de toute manière superflues [1]. Des chiffres invérifiables qui reposent sur des «experts» anonymes et des sources inconnues. Savoir quelles prestations seraient superflues ou pourquoi elles ne seraient plus fournies en cas de plafond budgétaire n'est pas précisé.

Actuellement, des experts mandatés par le DFI évaluent les expériences des Pays-Bas et de l'Allemagne en matière de plafond budgétaire [3]. Les données publiées par l'OCDE [4] (cf. fig.) montrent que les Pays-Bas n'en retirent aucun avantage en termes de coûts et que leur évolution y est même moins favorable. L'Allemagne, après 30 ans, ne mesure aucune économie par rapport à la Suisse, mais les prises en charge sont touchées, comme en témoigne un généraliste allemand [5]. L’Allemagne a ainsi dû créer en 2016 des centres uniquement pour référer les assurés nécessitant un rendez-vous urgent chez un spécialiste dans un délai de quatre semaines.

Conclusion: exiger plus du secteur ambulatoire tout en limitant les installations et les coûts ne peut pas fonctionner. C'est faire une politique des primes symbolique et non une politique de santé rationnelle, car un système de soins axé sur l'avenir requiert une médecine ambulatoire forte et donc un système de financement uniforme.

Crédits illustration

Graphique OCDE, légende FMH 